\title{
Demodex and Itching Ears
}

\author{
Carmen Antonela Bejinariu ${ }^{1,2}$, Eduard Vasile Nastase ${ }^{1,2 *}$, Felicia Trofin ${ }^{1,2}$, Ionela Larisa Miftode I $^{1,2}$ and \\ Olivia Simona Dorneanu ${ }^{1,2}$
}

${ }^{1}$ Grigore T Popa University of Medicine and Pharmacy, Iași, Romania

${ }^{2}$ Sf Parascheva Hospital of Infectious Diseases, Iași, Romania

*Corresponding author: Eduard Vasile Nastase, Grigore T Popa University of

Medicine and Pharmacy, Sf Parascheva Hospital of Infectious Diseases, Iași, Romania.

Received Date: February 17, 2021

Published Date: March 05, 2021

\begin{abstract}
While facial skin demodicosis was investigated during the time, ear demodicosis has only few but not to be neglected references. This ear disease should also be included on the list of the differential diagnosis in patients with damaged ears, especially when itching is one of the symptoms.

Keywords: Demodicosis; Demodex; Ears; Itching

Abbreviation: VAS: Visual analogue scale
\end{abstract}

\section{Introduction}

Demodicosis is a parasitic skin disease caused by Demodex folliculorum and Demodex brevis. Usually, D. folliculorum is found in the infundibular portion of the hair follicles, while D. brevis likes sebaceous ducts, Meibomian glands, and the deeper parts of hair follicles. The mites are translucent and worm-like with a head, four pairs of legs, and a longer body tail [1]. The lengths of adult mites reach $0.3-0.4 \mathrm{~mm}$ [2]. Their mainly nocturnal movements at a rate of $8-16 \mathrm{~mm} / \mathrm{h}$ [3] could explain why the itching is more annoying during this part of the day. The bright light determines the mites to hide into hair follicles [3], which could be an explanation to the reduced number of Demodex forms found when collecting the samples during the day. The meal of these mites includes a cocktail of skin cells, hormones and sebum accumulating in the hair follicles [3].

\section{Clinical Consideration}

There are two types of ear demodicosis: Demodectic frost of the ear and Auricular demodicosis [1]. Demodectic frost of the ear was described in an observation to a man in his 50s presenting for routine total body skin examination. He was completely asymptomatic, having frosted, gritty follicular scaling of his ears, superimposed on an erythematous base. Findings from skin scraping followed by potassium hydroxide preparation of the scale were positive for Demodex mites [3]. This demodectic frost of the ear presented fine follicular scaling primarily confined to the helix and lobule, giving the skin a frosted or powdery appearance and sandpaper-like texture. In some patients, there were also reported auricular erythema, pruritus, pain, and discomfort [4].

Auricular demodicosis was described in a case report on an 84-year-old woman, who presented herself for impaired hearing, but was found with chronic pruritic otitis externa, myringitis and a chronic cholesteatoma. Despite failing to establish the cause by microbiological and mycological investigations, histology of the external auditory canal and tympanic membrane revealed a large amount of Demodex mites [5].

In another study from Turkey, 50 enrolled patients were found with itching ears and on 54 health-control patients VAS was used to rate the itch, the itch period, and the medication used for itching. For the first group a positive and strongly significant relationship 
was observed between the number of Demodex spp. and severity of ear itch, with VAS scores of $\mathrm{p}=0.0001$ and $\mathrm{r}=0.724$ [6]. In another study performed in China on 613 college students, $11.58 \%$ were found positive with D. folliculorum and D. brevis. $67.60 \%$ from the positive cases complained constant scratching and itch [7]. As more than 5 mites/cm 2 are considered pathogenic for the patients with demodectic facial skin illness [8], in the examined studies included in our references we have not found any evidence about the pathogenic threshold number related to ears diseases caused by them.

\section{Discussion}

We found only few references in literature regarding to ear demodicosis, showing that it could be masked by others ear illness or mime them, as a possible reason for misdiagnosing the disease. The result may turn into a therapeutic failure and consequently a prolonged evolution of the disease. We highlight that (Figure 1) was taken from a microscopic examination of helix scales sample on a Romanian elderly male patient and was associated with the text to illustrate the observation about demodectic ear frost described. (Figure 2) was taken from a microscopic examination of a cerumen ear sample of another Romanian old man to illustrate the auricular demodicosis, even if the sampling method is different (in the case report the histological sampling method was used).

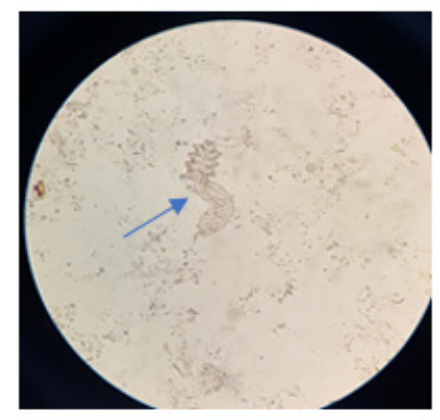

Figure 1: Demodex in helix scales (wet mount preparation with $20 \% \mathrm{KOH}, 10 \times$ )

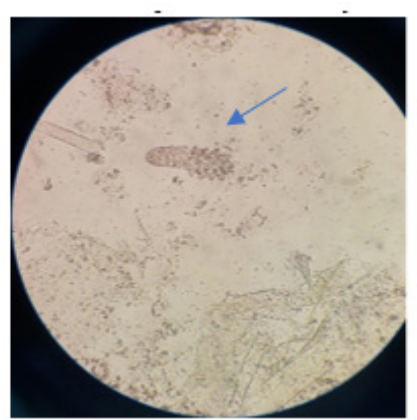

Figure 2: Demodex in cerumen (wet mount preparation with paraffin oil, 10x).

\section{Conclusion}

We recommend that parasitological examination should be a routine for any otologic investigation beside bacteriological and mycological procedures for patients presenting ear illness. We hope that this mini review will encourage the otorhinolaryngologists and dermatologists to pay attention on auricular demodicosis as a differential diagnostic, conducting their own research and publishing new and helpful findings.

\section{Acknowledgement}

The authors are grateful to Teodora Vremeră, MD, National Institute of Public Health, Bucharest, Romania, and Suzana Haivas, MD, SC Medical Center SRL, Dorohoi, Romania, for their agreement to share with us the photos performed by themself (Figure 1, Figure 2 , respectively), suggestive for the findings described in this mini review.

\section{Conflict of Interest}

The authors declare that they have no conflict of interest.

\section{Disclosure}

We have not found recent (in the last two years) articles related to the ear demodicosis. On the other hand, some of the original articles cited by the authors listed below in their paper works could not be found, as the reason for not quoting the primary source.

\section{References}

1. Fatimatuzzahra Al Haddar, Yudha Nurdian (2017) Demodectic Frost of the Ear, the New Presentation of Auricular Demodicosis: a Distinct Clinical Entity.

2. Rufli T, Mumcuoglu Y (1981) The hair follicle mites Demodex folliculorum and Demodex brevis: Biology and medical importance. A review. Dermatologica 162: 1-11.

3. Parvaiz Anwar Rather, Iffat Hassan (2014) Human Demodex Mite: The Versatile Mite of Dermatological Importance. Indian J Dermatol 59(1): 60-66.

4. Wallace Matthew M, Guffey Darren J, Wilson Barbara B (2017) Demodectic Frost of the Ear. JAMA Dermatol 153(3): 356-357.

5. Klemm E, Haroske G, Wollina U (2009) Otitis externa and myringitis due to demodicidosis. Acta Dermatovenerol Alp Panonica Adr 18(2): 73-76.

6. Bilal Nagihan, Kirişci Özlem, Özkaya Esra (2017) Demodex Species Infestation in Patients with Ear Itching and Its Relationship to Itch Severity. Turkiye Parazitol Derg 41(2): 87-91.

7. Ding Y, Huang X (2005) Investigation of external auditory meatus secretion Demodex folliculorum and Demodex brevis infection in college students. Lin Chuang Er Bi Yan Hou Ke Za Zhi 19(4): 176-177.

8. Așkın Ü, Seçkin D (2010) Comparison of the two techniques for measurement of the density of Demodex folliculorum: standardized skin surface biopsy and direct microscopic examination. BJD 162(5): 11241126. 